\title{
A variação da diversidade de microcrustáceos (Cladocera e Copepoda) a jusante de pequenos reservatórios é influenciada por táxons litorâneos
}

\author{
Milena Sartori' (1), Bárbara A. Martins² (1) \& Gilmar Perbiche-Neves' (1)
}

\author{
1. Laboratório de Plâncton, Departamento de Hidrobiologia, Universidade Federal de São Carlos, São Carlos, SP, Brasil. \\ (milena.sartori@gmail.com; gilmarperbiche83@gmail.com) \\ 2. Departamento de Zoologia, Universidade Estadual Paulista, Instituto de Biociências, campus de Botucatu, Botucatu, SP, Brasil. (barbara.a.martins@hotmail.com)
}

Recebido 7 agosto 2020

Aceito 14 janeiro 2021

Publicado 10 março 2021

DOI 10.1590/1678-4766e2021004

ABSTRACT. The variation of microcrustaceans diversity downstream of small reservoirs is influenced by litoranean taxa. The hypotheses tested were that, as well as in reservoirs of large surface areas, volumes and flow, in small reservoirs there is also a gradual and continuous dilution of the richness, diversity and abundance of microcrustaceans (Cladocera and Copepoda) downstream reservoirs, without the recomposition of richness downstream due to the lack of tributary rivers, marginal lagoons and even the small size of the stream, with no conditions for the development of its own potamoplankton. Two small reservoirs were studied and in their downstream stretches, with daily collections every six hours at eight sampling points, two lentic and six lotic downstream from each. Nineteen species were found (11 from Cladocera and eight from Copepoda), with emphasis on the greater number of coastal species than pelagic ones, and the greater abundance of coastal cladocerans at the points downstream of the reservoirs. There was no difference between the times, indicating that the spatial scale was more important than the temporal scale. There were higher values of ecological attributes in lentic environments, but there were significant increases in richness and diversity in the last points of the stretches of the stream, while the abundance decreased gradually downstream. No significant correlations were found for the abundance of taxa with any physical and chemical variable (temperature, $\mathrm{pH}$, dissolved oxygen, turbidity, total dissolved solids and transparency), indicating little or no effect of these variables. The cluster analysis with Euclidean distance separated two large groups, the first formed by the stretch downstream of the first reservoir and the second with the other points. In addition to the presence of litoranean taxa coming from reservoirs, there was an increase in litoranean taxa resident in streams. It can be concluded that there was a dilution of the abundance of species downstream of the streams, but the richness and diversity remained stable due to the contribution of litoranean taxa.

KEYWORDS. Zooplankton, spatial variation, recomposition, flow.

RESUMO. Testaram-se as hipóteses de que, assim como em reservatórios de grande áreas de superfície, volumes e vazão, em reservatórios pequenos também ocorrem a diluição gradual e contínua da riqueza, da diversidade e abundância de microcrustáceos (Cladocera e Copepoda) a jusante dos mesmos, sem a recomposição da riqueza a jusante pela falta de rios tributários, lagoas marginais e até pelo pequeno porte do riacho, sem condições para o desenvolvimento do potamoplâncton próprio do sistema. Foram estudados dois pequenos reservatórios e seus trechos a jusante, com coletas diárias a cada seis horas em oito pontos de amostragem, sendo dois lênticos e seis lóticos a jusante de cada. Foram encontradas 19 espécies (11 de Cladocera e oito de Copepoda), com destaque para o maior número de espécies litorâneas do que pelágicas, e a maior abundância de cladóceros litorâneos nos pontos a jusante dos reservatórios. Não houve diferença entre os horários, indicando que a escala espacial foi mais importante que a temporal. Houve maiores valores dos atributos ecológicos nos ambientes lênticos, porém houve aumentos significativos de riqueza e diversidade nos últimos pontos dos trechos de riacho, enquanto que a abundância diminuiu gradativamente a jusante. Não foram encontradas correlações significativas da abundância dos táxons com nenhuma variável limnológica (temperatura, $\mathrm{pH}$, oxigênio dissolvido, turbidez, sólidos totais dissolvidos e transparência), indicando pouco ou nenhum efeito destas variáveis. A análise de cluster com distância euclidiana separou dois grandes grupos, o primeiro formado pelo trecho a jusante do primeiro reservatório e o segundo com os demais pontos. Além da presença dos táxons litorâneos vindos dos reservatórios, houve incremento de táxons litorâneos residentes nos riachos. Pode-se concluir que houve uma diluição da abundância das espécies a jusante dos riachos, mas a riqueza e a diversidade permaneceram estáveis devido à contribuição de táxons litorâneos.

PALAVRAS-CHAVE. Zooplâncton, variação espacial, recomposição, vazão.

Nas últimas décadas, entre os ambientes aquáticos, os rios estão entre os ecossistemas naturais mais ameaçados do mundo, principalmente devido ao represamento dos seus cursos (NiLsson et al., 2005; Zhou et al., 2008; CZERniaWSKi \& KowalsKa-GóralsKa, 2018). Uma das principais consequências da construção de reservatórios é a quebra abrupta da paisagem hídrica, formando lagos artificiais conectados por rios em um sentido a jusante (SERAFIM-JÚNIOR et al., 2016). Um ecossistema lótico é transformado em um sistema lêntico, que acaba por alterar seus processos ecológicos como produção de matéria orgânica e ciclagem de nutrientes, modificando assim a estrutura das comunidades (LIERMANN et al., 2012; SERAFIMJÚNIOR et al., 2016). 
Em reservatórios que são utilizados para irrigação agrícola, abastecimento público, ou geração de energia elétrica, ocorrem uma notável variação do nível da água, podendo ser rápida e brusca, causando alterações nas descargas de água a jusante dos reservatórios (NALIATO et al., 2009), e mudanças nas comunidades planctônicas (MATSuURa et al., 2015; Portinho et al., 2016; CZERNiAWSKI \& KoWALSKA-GÓRALSKA, 2018).

A riqueza e a composição do zooplâncton podem ser afetadas pelo tamanho do reservatório (LONGATO et al., 2018), pela entrada de rios tributários (PORTINHO et al., 2016) e o pelo seu regime operacional, formato e profundidade (Perbiche-Neves \& Nogueira, 2013), fitoplâncton e variáveis físicas e químicas da água (ZHOU et al., 2008; Wu et al., 2010).

A alteração contínua do nível e da velocidade da água altera a dispersão do zooplâncton (MiTsUKA \& HENRY, 2002; PortinHo et al., 2016). A maior parte desses organismos tem grande potencial de dispersão, especialmente de maneira passiva, com seus ovos em repouso usando vários vetores como fluxo de água, pássaros, peixes e vento. As vias de dispersão por terra e por cursos de água são igualmente importantes para o zooplâncton, pois permitem que os organismos rastreiem mudanças ambientais (DECLERCK et al., 2011; VERBEEK et al., 2018). A limitação de dispersão ambiental é considerada uma dos principais fatores que estruturam as comunidades aquáticas, e as espécies geralmente têm potenciais diferentes de dispersão (BEISNER et al., 2006; DE BIE et al., 2012 ). Além de a dispersão ser alterada, pode ocorrer a presença de espécies diferentes que não são provenientes da dispersão, mas sim carreadas da fauna litorânea (SERAFIM-JÚNIOR et al., 2019).

Estudos realizados em trechos a jusante de reservatórios de grande área na bacia do Rio Paraná, como Jurumirim $\left(450 \mathrm{~km}^{2}\right)$ e Itaipu $\left(1250 \mathrm{~km}^{2}\right)$, indicam constante e gradual diluição do zooplâncton em distâncias entre 10 e 20 quilômetros a jusante das barragens, nos trechos lóticos (Mitsuka \& Henry, 2002; Portinho et al., 2016). O mesmo ocorre para o fitoplâncton em Itaipu (MATSUURA et al., 2015). Observa-se o acréscimo de táxons transportados por rios tributários e lagoas marginais, com reflexo no restabelecimento da riqueza a jusante em trechos lóticos, embora em baixa densidade (PorTINHo et al., 2016). Em outros países os resultados são similares, indicando também ampla predação do zooplâncton por peixes (AKOPIAN et al., 1999; DoI et al., 2008).

Contudo, os sistemas de menor tamanho de área, por exemplo, menores que $1 \mathrm{~km}^{2}$, são pouco estudados quanto à dinâmica do zooplâncton que é exportado a jusante. Nesses ambientes ZHOU et al. (2008) indicaram forte redução da densidade nos trechos lóticos logo após a região lêntica da barragem de um pequeno reservatório de baixo tempo de retenção de água na China, porém com pouca influência sobre a riqueza. No Brasil desconhecem-se os efeitos de pequenos reservatórios (açudes usados para irrigação) de água sobre o zooplâncton exportado a jusante, tornando-se o objetivo do presente estudo.
Testaram-se as seguintes hipóteses: (1) ocorre efeito de diluição da riqueza e da abundância dos microcrustáceos nos trechos a jusante dos pequenos reservatórios, mesmo com pequena vazão, sendo um padrão comum para estas regiões a jusante, e (2) a riqueza não será recomposta devido à ausência de rios tributários e de ambientes lênticos marginais nos trechos estudados, sendo um sistema de pequeno porte.

\section{MATERIAL E MÉTODOS}

Área de estudo. O estudo foi realizado em um trecho de uma pequena bacia hidrográfica de aproximadamente $15 \mathrm{~km}^{2}$, parte dela dentro do campus Lagoa do Sino da Universidade Federal de São Carlos, Buri, São Paulo, Brasil (Fig. 1). No trecho selecionado foram distribuídos oito pontos de amostragem em dois conjuntos de quatro pontos, sendo em cada um deles um ponto próximo dentro de cada reservatório (região lêntica) e três a jusante em cada um dos dois trechos de riachos, equidistantes entre si (Fig. 1). Considerando a distância via curso de rio, o primeiro gradiente estendeu-se por 1.600 metros a partir do lago 1 e o segundo por 430 metros, a partir do lago 2. O desnível total de altitude entre o ponto 1-L ao ponto $2-3$ foi de 8 metros.

No lago $1\left(40.200 \mathrm{~m}^{2}\right)$ a profundidade no local de coleta foi de dois metros, e no lago $2\left(15.200 \mathrm{~m}^{2}\right)$ foi de três metros. A média da vazão de água entre os pontos de amostragem foi de $27 \pm 8$ litros por segundo. Em ambos os lagos há bancos de macrófitas aquáticas submersas, com dominância de Egeria sp. (Hydrocharitaceae). Nos trechos de riacho a jusante dos lagos a profundidade variou entre 10 e 20 centímetros, e a largura destes trechos não foi superior a um metro.

Amostragens. As coletas nos oito pontos de amostragem foram realizadas em ciclos nictemerais a cada seis horas, sendo: 6:00h, 12:00h, 18:00h e 00:00h, durante dois dias consecutivos em outubro de 2018, totalizando duas coletas em cada horário. Não houve precipitações durante as coletas.

O zooplâncton foi coletado com uma rede cônica de 68 $\mu \mathrm{m}$ de abertura de malha. Nos riachos foram filtrados $300 \mathrm{~L}$ de água da superfície por amostra com um balde graduado de 20 L. Nos lagos foram realizados arrastos verticais com a rede de plâncton do fundo até a superfície (amostras integradas), e o volume filtrado (211 litros em ambos os lagos) foi obtido através da fórmula do volume do cilindro $\left(\mathrm{V}\right.$ cil $\left.=\pi \cdot \mathrm{r}^{2} . \mathrm{H}\right)$, considerando o raio de 15 centímetros da boca da rede e $\mathrm{H}$ como a altura do arrasto. No lago 1 foi feito 1,5 arrasto de 2 $\mathrm{m}$ de extensão, e no lago 2 um arrasto de $3 \mathrm{~m}$. Utilizando a mesma metodologia foram obtidas 64 amostras para análises quantitativas e 64 amostras para qualitativas, totalizando 128 amostras.

Concomitantemente às amostras de zooplâncton, foram mensuradas algumas variáveis ambientais com uma sonda multiparâmetros Hanna HI 98194 (Romênia): temperatura da água, turbidez, sólidos totais dissolvidos, condutividade elétrica, oxigênio dissolvido e pH. Através de um disco de Sechi foi mensurada a transparência da água (nos períodos noturnos usou-se uma lanterna para a visualização) e a profundidade com uma trena. 


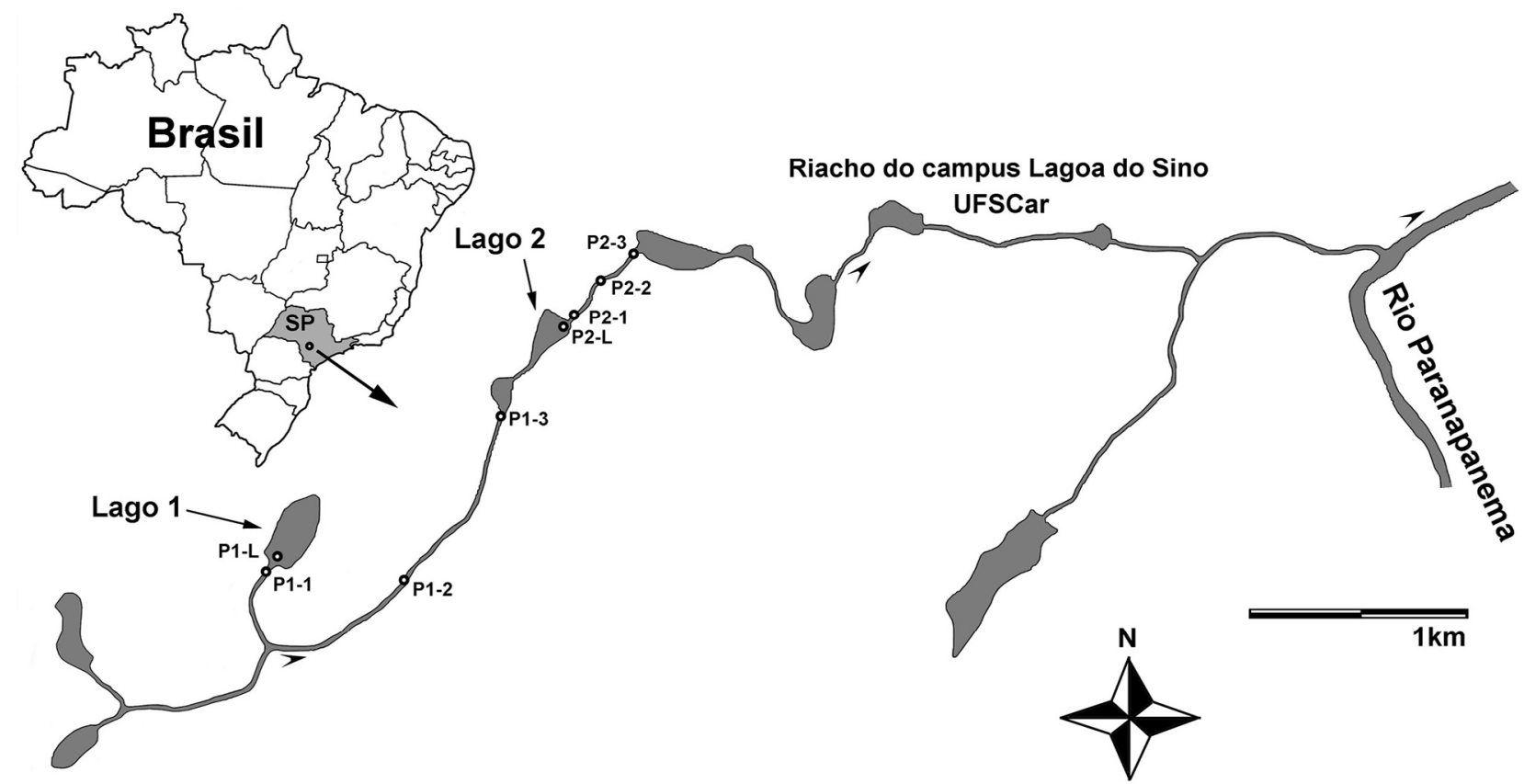

Fig. 1. Localização da área de estudo no Estado de São Paulo, Brasil e da bacia hidrográfica estudada, com os reservatórios (Lago 1 e Lago 2) e os trechos a jusante de cada um deles, com três pontos de amostragem.

Análises laboratoriais. As amostras foram triadas sobre estereomicroscópio Zeiss Stemi 305 e os organismos zooplanctônicos identificados com uso de microscópio binocular Axioscope no menor nível taxonômico possível utilizando bibliografia especializada (e.g. ReID, 1985; Elmoor-Loureiro, 1997; Perbiche-Neves et al., 2015; ElmoOr-Loureiro, 2020). As contagens das amostras foram feitas com cubetas de acrílico em estereoscópio para cladóceros, copepoditos (identificados ao nível de ordem) e copépodes adultos, sendo quantificados ao menos 200 indivíduos por amostra ou a amostra toda no caso de poucos indivíduos. Náuplios de copépodes, identificados também ao nível de ordem, foram quantificados em câmara de Sedgewick-Rafter através de subamostragens de $1 \mathrm{~mL}$ até atingir 100 indivíduos por amostra, sob microscópio óptico Zeiss Primo Star. Entre os atributos ecológicos foram calculadas a riqueza específica, por meio da soma total de espécies, e a abundância dos organismos, expressos em indivíduos por metro cúbico, pela fórmula:

Abundância $=\mathrm{A} / \mathrm{V} * 1000$, onde $\mathrm{A}$ = número de indivíduos contados na amostra e $\mathrm{V}=$ volume de água filtrado na coleta.

Análise dos dados. Após a logaritimização dos dados foram realizados testes de normalidade (ShapiroWilk), e como os dados apresentaram-se não paramétricos, procedeu-se com teste de Kruskal-Wallis $(\mathrm{p}<0,05)$ para comparar os valores das variáveis limnológicas e dos atributos ecológicos entre os pontos e horários, com teste a posteriori de Dunn $(\mathrm{p}<0,05)$ para verificar grupos homogêneos entre os pontos e horários. Pelo fato de a transparência ter sido quase máxima em todas as amostragens (ambientes rasos) e ter estado relacionada com a profundidade, essa variável não foi comparada estatisticamente.

Para correlacionar as abundâncias das espécies zooplanctônicas e as variáveis limnológicas foi aplicada uma análise de correspondência canônica (CCA) com 1000 permutações e nível de confiança 0,05 (95\%), usando o pacote vegan 2.3.5 para o R (OKSANEN et al., 2013). Táxons com baixa ocorrência e abundância e formas imaturas foram removidos desta análise.

Análise hierárquica de cluster com distância euclidiana foi utilizada para agrupar pontos de amostragem similares quanto à abundância dos táxons. Fizeram-se médias entre os horários de cada ponto. Todas as análises foram realizadas no software R Cran Project 3.4.4 (2019).

\section{RESULTADOS}

Variáveis limnológicas. Todas as variáveis limnológicas apresentaram diferença significativa entre os pontos de amostragem, porém apenas o $\mathrm{pH}$ entre os horários (Tab. I). A temperatura da água e o oxigênio dissolvido foram maiores no reservatório 2 , enquanto que as demais ( $\mathrm{pH}$, condutividade elétrica, sólidos totais dissolvidos, turbidez e transparência) no reservatório 1 (Tab. II). De modo geral houve maiores valores de temperatura, sólidos totais dissolvidos, turbidez, transparência e de condutividade nos pontos lênticos, com decréscimos a jusante. Para o oxigênio dissolvido foi o contrário, com menores valores nos reservatórios. Destaca-se também os maiores valores de condutividade, sólidos totais dissolvidos e turbidez no reservatório 1 . A tendência de variação do $\mathrm{pH}$ identificada pelo teste de Dunn entre os horários indicou maior acidez durante a noite ( $18: 00$, média $=4,7 ; 00: 00$, média $=4,8 ; 06: 00$, média $=4,7)$ e menor acidez ao meio dia (12:00, média=5,1). 
Tab. I. Resultados do teste de Kruskal-Wallis (KW) comparando variáveis limnológicas entre os pontos e os horários amostrados. Em negrito, as diferenças significativas $(\mathrm{p}<0,05)$ pelo teste de KW.

\begin{tabular}{lcccc}
\hline & & Pontos & & Horas \\
& Chi & p & Chi & 0,35 \\
\hline Temperatura $\left({ }^{\circ} \mathrm{C}\right)$ & 28,48 & $\mathbf{0 , 0 0}$ & 4,4 & $\mathbf{0}, \mathbf{0 2}$ \\
pH & 22,42 & $\mathbf{0 , 0 0}$ & 9,21 & 0,27 \\
Oxigênio Dissolvido $\left(\mathrm{mg} . \mathrm{L}^{-1}\right)$ & 36,79 & $\mathbf{0 , 0 0}$ & 5,1 & 0,07 \\
Condutividade elétrica $\left(\mu \mathrm{S} . \mathrm{cm}^{-1}\right)$ & 18,79 & $\mathbf{0 , 0 0}$ & 8,46 & 0,06 \\
STD $\left(\mathrm{mg} . \mathrm{L}^{-1}\right)$ & 19,35 & $\mathbf{0 , 0 0}$ & 9,36 & 0,87 \\
Turbidez $(\mathrm{NTU})$ & 29,69 & $\mathbf{0 , 0 0}$ & 1,23 & 0,82 \\
Transparência $(\mathrm{cm})$ & 54,48 & $\mathbf{0 , 0 0}$ & 1,52 & \\
\hline
\end{tabular}

Tab. II. Valores médios das variáveis limnológicas entre os pontos de amostragem no presente estudo (O. D., oxigênio dissolvido; Cond., condutividade; STD, sólidos totais dissolvidos). Letras indicam grupos homogêneos $(\mathrm{p}<0,05)$ pelo teste a posteriori de Dunn, sobre o teste de Kruskal-Wallis.

\begin{tabular}{lccccccc}
\hline & Temp. & $\mathrm{pH}$ & O. D. & Cond. & STD & Turbidez & Transparência \\
\hline P1-L & $21,56 \pm 1 \mathrm{a}$ & $5,6 \pm 0,2 \mathrm{a}$ & $3,21 \pm 1,2 \mathrm{a}$ & $34,88 \pm 8,6 \mathrm{a}$ & $17,50 \pm 6,1 \mathrm{a}$ & $4,92 \pm 0,5 \mathrm{a}$ & $113,75 \pm 11$ \\
P1-1 & $19,99 \pm 0,3 \mathrm{~b}$ & $5,2 \pm 0,3 \mathrm{~b}$ & $3,54 \pm 0,5 \mathrm{a}$ & $17,75 \pm 4,3 \mathrm{~b}$ & $6,75 \pm 2,02 \mathrm{~b}$ & $1,35 \pm 1,3 \mathrm{~b}$ & $7,75 \pm 0,3$ \\
P1-2 & $20,50 \pm 0,5 \mathrm{~b}$ & $5,0 \pm \mathrm{b}$ & $5,47 \pm 0,7 \mathrm{~b}$ & $17,00 \pm 8 \mathrm{~b}$ & $8,17 \pm 3,8 \mathrm{~b}$ & $5,38 \pm 2,6 \mathrm{a}$ & $30,17 \pm 1,5$ \\
P1-3 & $20,36 \pm \mathrm{b} 1,3$ & $5,1 \pm 1 \mathrm{~b}$ & $7,72 \pm 1,8 \mathrm{c}$ & $29,71 \pm 27,7 \mathrm{a}$ & $6,75 \pm 2 \mathrm{~b}$ & $1,28 \pm 0,3 \mathrm{~b}$ & $38,38 \pm 0,7$ \\
P2-L & $23,44 \pm 1 \mathrm{c}$ & $4,7 \pm 0,2 \mathrm{c}$ & $5,96 \pm 0,6 \mathrm{~b}$ & $14,88 \pm 3,1 \mathrm{c}$ & $7,63 \pm 1,6 \mathrm{~b}$ & $4,20 \pm 3,1 \mathrm{a}$ & $2,00 \pm 0$ \\
P2-1 & $22,90 \pm 1,1 \mathrm{~d}$ & $4,5 \pm 0,1 \mathrm{c}$ & $7,28 \pm 0,6 \mathrm{c}$ & $13,13 \pm 2,1 \mathrm{c}$ & $6,88 \pm 1,1 \mathrm{~b}$ & $2,13 \pm 0,3 \mathrm{c}$ & $16,00 \pm 2,5$ \\
P2-2 & $23,15 \pm 0,7 \mathrm{~d}$ & $4,6 \pm 0,2 \mathrm{c}$ & $7,22 \pm 0,4 \mathrm{c}$ & $12,88 \pm 3,6 \mathrm{c}$ & $7,13 \pm 1,6 \mathrm{~b}$ & $2,61 \pm 0,5 \mathrm{c}$ & $33,88 \pm 3,3$ \\
P2-3 & $22,66 \pm 0,8 \mathrm{~d}$ & $4,6 \pm 0,1 \mathrm{c}$ & $6,94 \pm 0,6 \mathrm{c}$ & $13,50 \pm 3,2 \mathrm{c}$ & $6,63 \pm 1,9 \mathrm{~b}$ & $2,88 \pm 0,2 \mathrm{c}$ & $28,38 \pm 1,2$ \\
\hline
\end{tabular}

Microcrustáceos. Foram registradas 19 espécies (11 de Cladocera e oito de Copepoda) (Tab. III). Entre as 11 espécies de Cladocera duas foram exclusivas de ambiente lêntico (Ceriodaphnia silvestrii Daday, 1902 e Diaphanossoma birgei Korinek, 1981) e duas exclusivas de ambiente lótico [Macrothrix paulensis (Sars, 1900) e Macrothrix spinosa King, 1853]. Quanto ao tipo de hábitat, cinco espécies foram classificadas como litorâneas, cinco pelágicas e uma nos dois habitats (Tab. III). Entre as oito espécies de Copepoda apenas uma foi exclusiva do ambiente lêntico (Eucyclops prionophorus Kiefer, 1931) e uma do lótico [Microcyclops ceibaensis (Marsh, 1919)]. Quatro espécies foram litorâneas, uma pelágica e três de ambos hábitats (Tab. III).

A riqueza de espécies, a diversidade alfa com o índice de Shannon-Wienner (H'), a abundância total e a abundância da maioria dos táxons apresentaram diferenças entre os pontos de amostragem (Tab. IV).

Os maiores valores de diversidade (H') e de abundância total foram encontrados nas regiões lênticas de ambos os reservatórios, entretanto para a riqueza houve maior valor apenas na região lêntica do reservatório 1, enquanto que no reservatório 2 houve valores menores do que no seu trecho a jusante (Figs 3, 4).
Diferente da abundância total, para a riqueza e diversidade houve aumento nos pontos 2 e 3 (Figs 2, 3 ), indicando recomposição destes valores a jusante dos reservatórios especialmente pela presença de cladóceros litorâneos (Fig. 6), diferente dos cladóceros pelágicos (Fig. 5) e copépodes (Figs 7,8).

A CCA explicou $76 \%$ da variação dos dados $(41 \%$ na primeira variável canônica e $35 \%$ na segunda) (Tab. V), porém nenhuma variável limnológica apresentou correlação significativa, sugerindo ausência de efeitos destas sobre a abundância dos microcrustáceos.

A análise de cluster (Fig. 9) com distância euclidiana agrupou os pontos de amostragem quanto à abundância, considerando que não houve diferenças significativas entre os horários. Os resultados foram similares com os verificados com o teste de Kruskal-Wallis para a abundância dos táxons. O trecho de riacho a jusante do reservatório 1 (pontos P1-1, P1-2, P1-3) formou um agrupamento homogêneo, enquanto que os demais pontos formaram outro grande grupo: os pontos P2-2 e P2-3 com o ponto lêntico no reservatório 2 (P2-L), e o ponto lêntico no reservatório 1 (P1-L) assemelhou-se com o primeiro ponto lótico após o reservatório 2 (P2-1). 
Tab. III. Lista de espécies registradas no presente estudo, representadas nos ambientes lênticos (dois lagos) e trechos de riacho (lótico), e ainda o tipo de hábitat de cada táxon de acordo com SERAFIM-JúNior et al. $(2002,2016)$.

\begin{tabular}{|c|c|c|c|}
\hline Táxon & Lêntico & Lótico & Habitat \\
\hline \multicolumn{4}{|l|}{ Cladocera } \\
\hline Acroperus tupinamba Sinev \& Elmoor-Loureiro, 2010 & $\mathrm{X}$ & $\mathrm{X}$ & litorâneo \\
\hline Bosminopsis deiters Richard, 1834 & $\mathrm{X}$ & $\mathrm{X}$ & pelágico \\
\hline Ceriodaphnia cornuta Sars, 1886 & $\mathrm{X}$ & $\mathrm{X}$ & pelágico \\
\hline Ceriodaphnia silvestrii Daday, 1902 & $\mathrm{X}$ & & pelágico \\
\hline Diaphanosoma birgei Korineck, 1981 & $\mathrm{X}$ & & pelágico \\
\hline Diaphanosoma fluviatile Hansen, 1899 & $\mathrm{X}$ & $\mathrm{X}$ & pelágico \\
\hline Ilyocryptus spinifer Herrick, 1882 & $\mathrm{X}$ & $\mathrm{X}$ & litorâneo \\
\hline Macrothrix laticornis (Jurine, 1820) & $\mathrm{X}$ & $\mathrm{X}$ & litorâneo \\
\hline Macrothrix paulensis (Sars, 1900) & & $\mathrm{X}$ & litorâneo \\
\hline Macrothrix spinosa King, 1853 & & $\mathrm{X}$ & litorâneo \\
\hline Simocephalus latirostris (Stingelin, 1906) & $\mathrm{X}$ & $\mathrm{X}$ & litorâneo/pelágico \\
\hline \multicolumn{4}{|l|}{ Copepoda } \\
\hline Argyrodiaptomus furcatus (Sars, 1901) & $\mathrm{X}$ & $\mathrm{X}$ & pelágico \\
\hline Eucyclops prionophorus Kiefer, 1931 & $\mathrm{X}$ & & litorâneo \\
\hline Mesocyclops meridianus (Kiefer, 1926) & $\mathrm{X}$ & $\mathrm{X}$ & litorâneo/pelágico \\
\hline Microcyclops anceps anceps (Richard, 1897) & $\mathrm{X}$ & $\mathrm{X}$ & litorâneo/pelágico \\
\hline Microcyclops ceibaensis (Marsh, 1919) & & $\mathrm{X}$ & litorâneo \\
\hline Microcyclops finitimus Dussart, 1984 & $\mathrm{X}$ & $\mathrm{X}$ & litorâneo \\
\hline Paracyclops chiltoni (Thomson, 1882) & $\mathrm{X}$ & $\mathrm{X}$ & litorâneo \\
\hline Mesocyclops longisetus longisetus (Thiébaud, 1914) & $\mathrm{X}$ & $\mathrm{X}$ & litorâneo/pelágico \\
\hline
\end{tabular}

Tab. IV. Resultados do teste de Kruskal-Wallis comparando os atributos ecológicos (riqueza, diversidade, abundância total e demais categorias de abundância) entre os pontos e os horários amostrados. Em negrito, as diferenças significativas $(\mathrm{p}<0,05)$.

\begin{tabular}{lcccc}
\hline & & Pontos & & Horas \\
& Chi & $\mathrm{p}$ & Chi & 0,24 \\
\hline Riqueza & 24,0 & $\mathbf{0 , 0 0}$ & 5,40 & 0,21 \\
Diversidade H' & 22,96 & $\mathbf{0 , 0 0}$ & 5,80 & 0,57 \\
Abundância total & 32,42 & $\mathbf{0 , 0 0}$ & 2,91 & 0,68 \\
Cladocera pelágicos & 39,3 & $\mathbf{0 , 0 0}$ & 2,28 & 0,71 \\
B. deitersi & 25,19 & $\mathbf{0 , 0 0}$ & 2,1 & 0,58 \\
S. latirostris & 24,18 & $\mathbf{0 , 0 0}$ & 2,85 & 0,60 \\
Cladocera litorâneos & 23,31 & $\mathbf{0 , 0 0}$ & 2,73 & 0,26 \\
M. spinosa & 12,21 & $\mathbf{0 , 0 9}$ & 5,18 & 0,77 \\
A. harpae & 19,37 & $\mathbf{0 , 0 0}$ & 1,78 & 0,48 \\
I. spinifer & 17,42 & $\mathbf{0 , 0 1}$ & 3,42 & 0,91 \\
Náuplios & 37,46 & $\mathbf{0 , 0 0}$ & 0,93 & 0,82 \\
Copepodito Calanoida & 10,39 & $\mathbf{0 , 1 6}$ & 0,62 \\
Copepodito Cyclopoida & 34,03 & $\mathbf{0 , 0 0}$ & 2,63 & \\
\hline
\end{tabular}

\section{DISCUSSÃO}

Os resultados indicam que em sistemas de pequeno porte (reservatórios com pequenos tamanhos de área e baixo fluxo de água), comparado por exemplo aos reservatórios de grandes usinas hidrelétricas, a riqueza e a diversidade encontrada nos trechos a jusante foram representadas principalmente por espécies litorâneas.
Assim, a hipótese 1 foi parcialmente aceita, pois houve aumento da riqueza e diversidade nos pontos a jusante, contudo foi verificada diminuição e o efeito de diluição da abundância. O padrão encontrado em sistemas maiores, com diluição ampla e gradual da abundância e riqueza de espécies a jusante (e.g. AKOPIAN et al., 1999; PorTinHo et al., 2016), não foi encontrado em sistemas pequenos, onde esse efeito só ocorreu para a abundância e não para a riqueza e diversidade. 

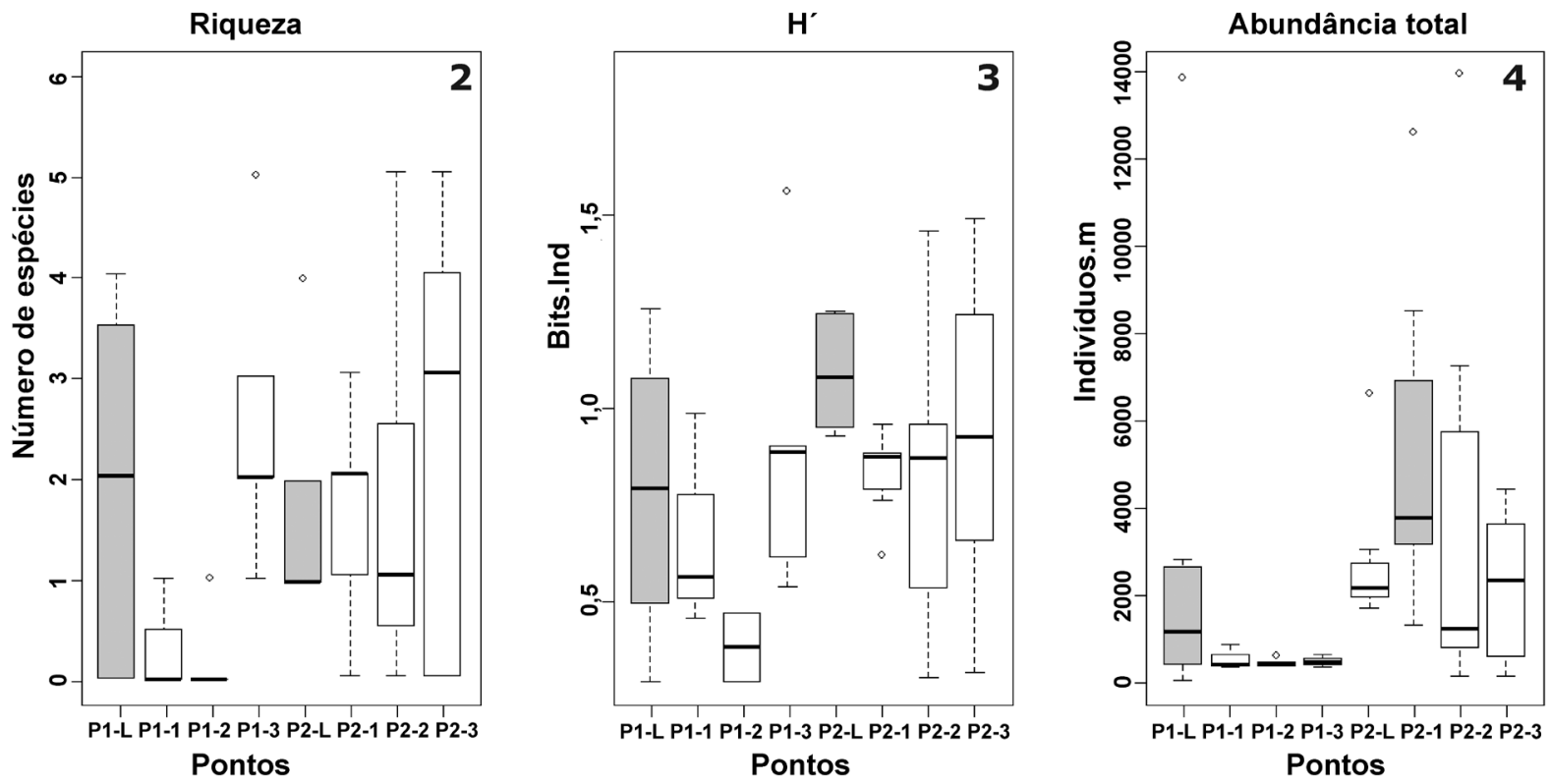

Figs 2-4. Boxplots com mediana e quartis dos atributos ecológicos que apresentaram diferença significativa no teste de Kruskal-Wallis entre os pontos de amostragem: riqueza (2), índice de diversidade de Shannon-Wienner $\left(\mathrm{H}^{\prime}\right)(3)$ e abundância total (4). Em cinza os pontos localizados nos reservatórios.
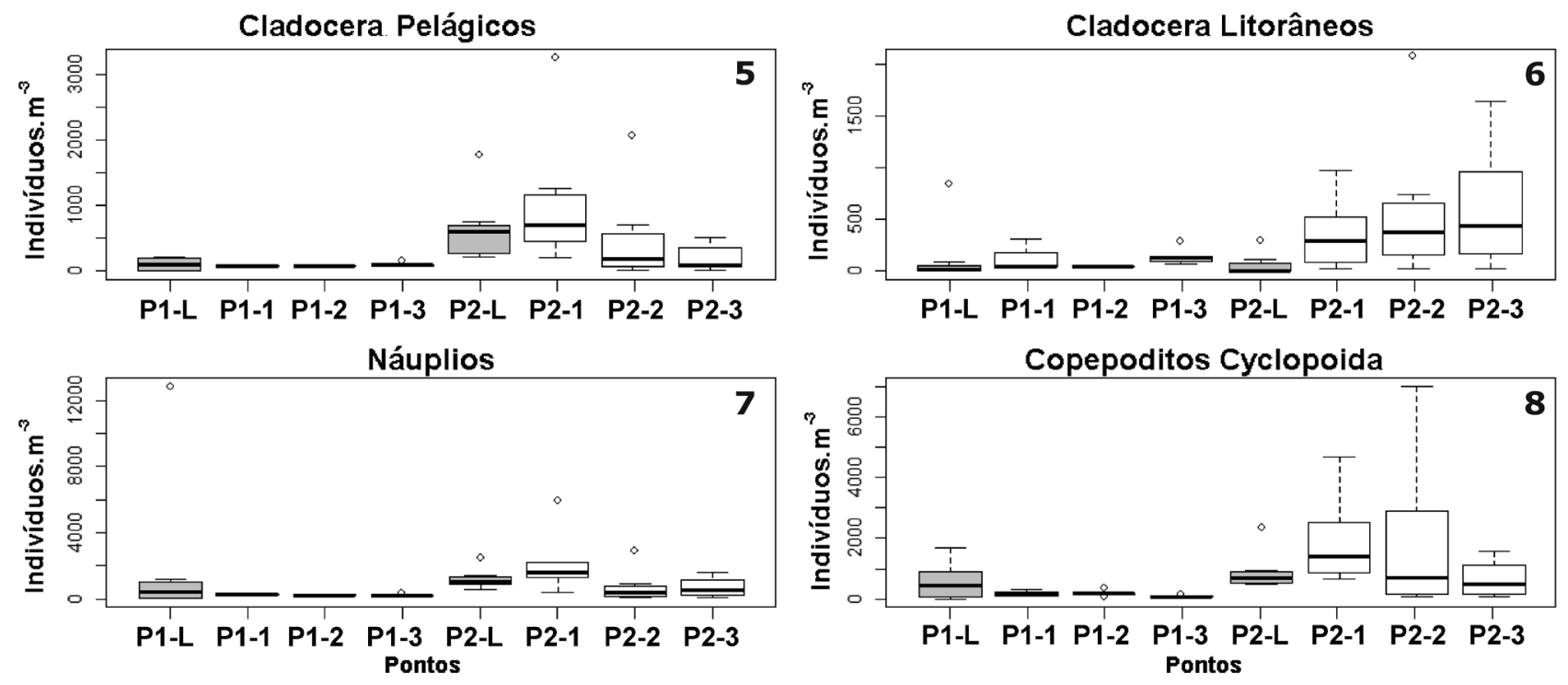

Figs 5-8. Boxplots com mediana e quartis dos valores de abundância dos táxons que apresentaram diferenças significativas no teste de Kruskal-Wallis entres os pontos de amostragem: Cladocera pelágicos (5), Cladocera limnéticos (6), náuplios (7) e copepoditos de Cyclopoida (8). Em cinza os pontos localizados nos reservatórios.

Tab. V. Resultados da análise de correspondência canônica usando 1000 permutações: escores das variáveis canônicas 1 e 2 (VC1, VC2), coeficiente de correlação canônica $\left(r^{2}\right)$ e valor de significância $(p)$.

\begin{tabular}{lcccc}
\hline & CA1 & CA2 & $\mathrm{r}^{2}$ & $\mathrm{p}$ \\
\hline Temperatura da água & $-0,77$ & 0,63 & 0,03 & 0,79 \\
pH & 0,98 & 0,17 & 0,04 & 0,71 \\
Oxigênio dissolvido & $-0,98$ & $-0,21$ & 0,12 & 0,31 \\
Condutividade elétrica & 0,17 & 0,99 & 0,05 & 0,55 \\
Turbidez & 0,88 & $-0,48$ & 0,20 & 0,11 \\
Transparência & $-0,64$ & 0,77 & 0,15 & 0,23 \\
Explicação & $41 \%$ & $35 \%$ & & \\
\hline
\end{tabular}




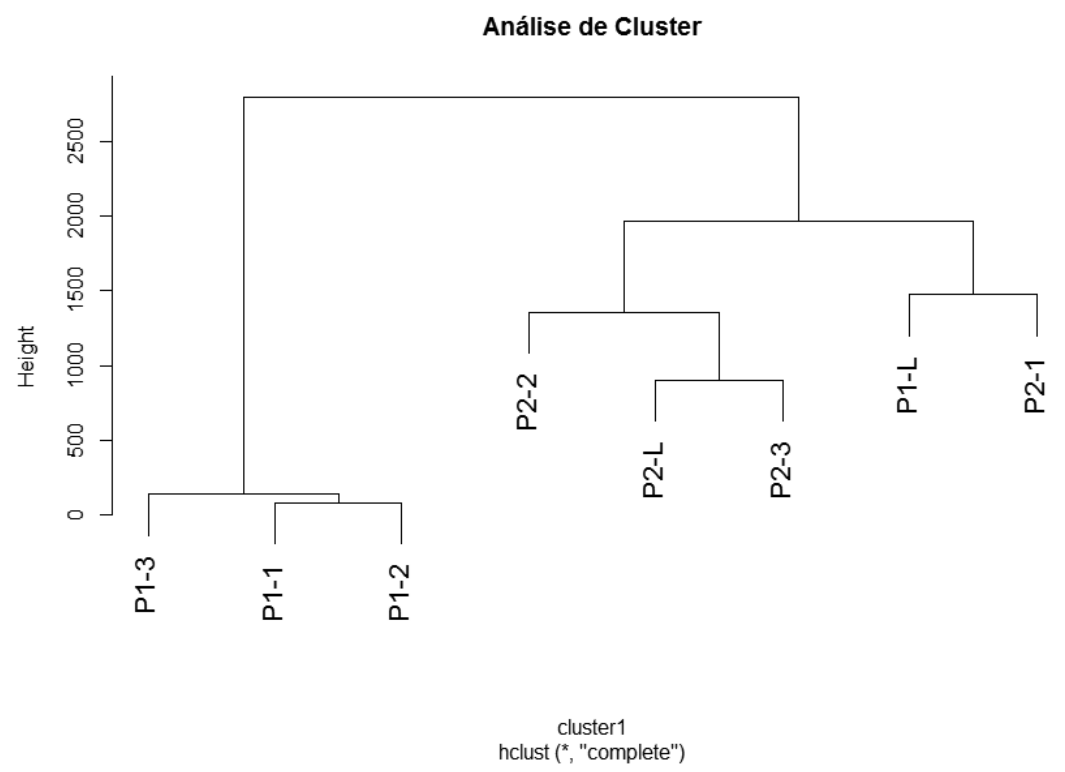

Fig. 9. Resultado da análise de cluster utilizando distância euclidiana para agrupar os pontos de amostragem quanto à abundância dos táxons.

Diferente dos ambientes de maior porte, onde há acréscimo e o aporte de diversidade pelos rios tributários e lagoas marginais, no presente caso, as espécies litorâneas residem no ambiente lótico, possivelmente pela baixa velocidade e vazão da água e também pela presença de hábitats que permitem que não sejam arrastadas pela correnteza. Nesse sentido a hipótese 2 foi rejeitada, pois mesmo sem rios tributários e lagoas marginais a riqueza e a diversidade foram recompostas, devido aos táxons litorâneos residentes nos ambientes a jusante.

A diminuição da abundância a jusante nos pontos lóticos se confirmou, e segundo a literatura, em parte, é apontada pela elevada correnteza (MitsuKa \& HenRY, 2002; Portinho et al., 2016; CZERNiAWSKI \& KowALSKAGóRALSKA, 2018) e pela predação de peixes planctívoros (AKopian et al., 1999; HAVENs et al., 2007; Dor et al., 2008).

Pelas diferenças detectadas entre os pontos lênticos dentro dos reservatórios e os pontos lóticos a jusante, confirma-se que o tempo de residência é um dos maiores fatores governantes da estrutura zooplanctônica nos ambientes aquáticos continentais, mesmo para sistemas de pequeno porte (ZHou et al., 2008). A baixa vazão da água favorece a retenção dos táxons nos ambientes lóticos, aumentando o tempo de residência do ambiente para os microcrustáceos e sua residência neste ambiente, em especial os táxons litorâneos, enquanto que para os pelágicos a redução continuou ocorrendo a jusante.

Embora as variáveis limnológicas tenham diferido entre os pontos, elas não exerceram influência sobre a distribuição dos microcrustáceos, conforme pode ser verificado na CCA. $\mathrm{O}$ fato de os ambientes estudados estarem localizados numa escala espacial pequena, dos reservatórios serem parecidos em termos de área, profundidade, formato e ainda conectados no sentido montante-jusante, podem ter favorecido o resultado de pouca influência sobre os organismos. Se comparados, por exemplo, a reservatórios de grande área, volume e profundidade, onde ocorre ampla compartimentalização espacial, as variáveis limnológicas tendem a exercer maior efeito sobre as assembleias de microcrustáceos, como temperatura, transparência, nutrientes e clorofila (Nogueira et al., 2008; Illyová \& PAStuchová, 2012; Perbiche-Neves \& Nogueira, 2013; Picapedra et al., 2020).

Conclui-se que os resultados contrastam parcialmente com os encontrados em reservatórios de grande área e volume, pois houve aumento da riqueza e da diversidade a jusante, mesmo sem a entrada de rios tributários ou conexões com lagoas marginais, ou ainda distância o suficiente para o desenvolvimento do potamoplâncton com elevada diversidade. Ressalta-se o pequeno tamanho da escala espacial, com baixo volume de água e pequena distância espacial. Verificou-se que as espécies litorâneas, especialmente de cladóceros, contribuem para elevados valores de riqueza e diversidade a jusante.

A diluição da abundância a jusante dos reservatórios corrobora os demais estudos, possivelmente devido ao aumento da correnteza e pela pressão de predação por peixes de pequeno porte.

No presente estudo, locais conectados, reservatórios pequenos e com baixa vazão, as variáveis limnológicas mensuradas não mostraram exercer forte efeito sobre os microcrustáceos nestes tipos de ambientes.

Agradecimentos. Ao PIBIC (Bolsa de Iniciação Científica de MS 146604/2018-0); ao M.Sc. André Pereira da Silva pelo auxílio no campo; ao Dr. Paulo H. F. Lucinda e aos revisores anônimos pelas valiosas contribuições no melhoramento do artigo. 


\section{REFERÊNCIAS}

AKopian, M.; Garnier J. \& Pourriot, R. 1999. A large reservoir as a source of zooplankton for the river: structure of the populations and influence of fish predation. Journal of Plankton Research 21(2):285-297.

Beisner, B. E.; Peres-Neto, P. R.; Lindström, E. S.; Barnett, A. \& LONGHI, M. L.

2006. The role of environmental and spatial processes in structuring lake communities from bacteria to fish. Ecology 87(12):2985-2991.

Czerniawski, R. \& Kowalska-Góralska, M. 2018. Spatial changes in zooplankton communities in a strong human-mediated river ecosystem. PeerJ 6:e5087.

De Bie, T. L.; De Meester, L.; Brendonck, K.; Martins, B.; Goddeeris, D.; Ercken, H.; Hampel, L.; Denys, L.; Vanhecke, K.; Van Der Gucht, J.; VAn Wichelen, W.; Vyverman, S. A. \& DeClerCK, J. 2012. Body size and dispersal mode as key traits determining metacommunity structure of aquatic organisms. Ecology Letters 15(7):740-747.

Declerck, S. A. J.; Coronel, J. S.; Legendre, P. \& Brendonck, L. 2011. Scale dependency of processes structuring metacommunities of cladocerans in temporary pools of High-Andes wetlands. Ecography 34(2):296-305.

Doi, H.; Chang, K. H.; Ando, T.; Imai, H.; NaKano, S. I.; KaJimoto, A. \& Katano, I. 2008. Drifting plankton from a reservoir subsidize downstream food webs and alter community structure. Oecologia 156(2):363-371.

ElmoOR-LouReIro. L. M. A. 1997. Manual de identificação de cladóceros límnicos do Brasil. Brasília, Universa. 156p.

Elmoor-Loureiro, L. M. A. 2020. Cladóceros do Brasil: Famílias Chydoridae e Eurycercidae. Available at $<$ https://cladocera.wordpress. com/>. Accessed on 07 August 2020.

Havens, K. E.; East, T. L. \& Beaver, J. R. 2007. Zooplankton response to extreme drought in a large subtropical lake. Hydrobiologia 589(1):187198.

ILLYOvÁ, M. \& PASTUChOvá, Z. 2012. The zooplankton communities of small water reservoirs with different trophic conditions in two catchments in western Slovakia. Limnologica 42(4):271-281.

LIERMANN, C. R.; NiLSSON, C.; ROBERTSON, J. \& NG, R. Y. 2012. Implications of Dam Obstruction for Global Freshwater Fish Diversity. Bioscience 62(6):539-548.

Longato, L. O.; Ferreira, I. E. P. \& Perbiche-Neves, G. 2018. Relações entre a riqueza do zooplâncton e a área em lagos brasileiros: comparando lagos naturais e artificiais e tendências. Acta Limnologica Brasiliensia 30:6.

Matsuura, P.; Perbiche-Neves, G.; Ferreira, R. A. R. \& Nogueira, M. G. 2015. Changes in the phytoplankton structure downstream a large reservoir: Effects of tributaries on the assemblages attributes. Biologia Section Botany 70:1-8.

Mitsuka, P. M. \& Henry, R. 2002. The fate of copepod populations in the Paranapanema River (São Paulo, Brazil), downstream from the
Jurumirim. Brazilian Archives of Biology and Technology 45(4):479490.

Naliato, D. A.; Nogueira, M. G. \& Perbiche-Neves, G. 2009: Discharge pulses of hydroelectric dams and their effects in the downstream limnological conditions: A case study in a large tropical river (SE Brazil). Lakes \& Reservoirs: Research and Management 14:301-314.

Nilsson, C.; Reidy, C. A.; Dynesius, M. \& Revenga, C. 2005. Fragmentation and flow regulation of the world's large river systems. Science 308(5720):405-408.

Nogueira, M. G.; Reis Oliveira, P. C. \& Britto, Y. T. 2008. Zooplankton assemblages (Copepoda and Cladocera) in a cascade of reservoirs of a large tropical river (SE Brazil). Limnetica 27(1):151-170.

OKsanen, J.; Blanchet, F. G.; Kindt, R.; Legendre, P.; Minchin, P. R.; O’Hara, R. B. \& OKsanen, M. J. 2013. Package 'vegan'. Community ecology package, version, 2(9): 1-295.

Perbiche-Neves, G. \& Nogueira, M. G. 2013. Reservoir design and operation: effects on aquatic biota: a case study of planktonic copepods. Hydrobiologia 707(1):187-198.

Perbiche-Neves, G.; Boxshall, G. A.; Previattelli, D.; Nogueira, M. G. \& DA Rocha, C. E. F. 2015. Identification guide to some Diaptomid species (Crustacea, Copepoda, Calanoida, Diaptomidae) of "de la Plata" River Basin (South America). ZooKeys 497(1):4-7.

Picapedra, P. H. S.; Fernandes, C.; Taborda, J.; Baumgartner, G. \& SANCHES, P. V. 2020. A long-term study on zooplankton in two contrasting cascade reservoirs (Iguacu River, Brazil): effects of interannual, seasonal, and environmental factors. PeerJ 8:e8979.

Portinho, J. L.; Perbiche-Neves, G. \& Nogueira, M. G. 2016. Zooplankton community and tributary effects in free-flowing section downstream a large tropical reservoir. International Review of Hydrobiology 101(1-2):48-56.

ReID, J. W. 1985. Chave de identificação e lista de referências bibliográficas para as espécies continentais sulamericanas de vida livre da ordem Cyclopoida (Crustacea, Copepoda). São Paulo, Instituto de Biociências da Universidade de São Paulo, p. 95-107.

Serafim-Júnior, M.; Lansac-Tôha, F. A.; Lopes, R. M. \& Perbiche-Neves, G. 2016. Continuity effects on rotifers and microcrustaceans caused by the construction of a downstream reservoir in a cascade series (Iguaçu River, Brazil). Brazilian Journal of Biology 76(2):279-291.

Serafim-Júnior, M.; Perbiche-Neves, G. \& LansaC-TôHa, F. A. 2019. An assessment of the factors determining rotifer assemblage in river-lake systems: the effects of seasonality and habitat. Zoologia 36:e24191.

VerbekK, L.; Gall, A.; Hillebrand, H. \& Striebel, M. 2018. Warming and oligotrophication cause shifts in freshwater phytoplankton communities. Global Change Biology 24(10):4532-4543.

Wu, N.; Tang, T.; Fu, X.; Jiang, W.; LI, F.; Zhou, S.; CaI, Q. \& Fohrer, N. 2010. Impacts of cascade run-of-river dams on benthic diatoms in the Xiangxi River, China. Aquatic Sciences 72(1):117-125

Zhou, S. C.; TANG, T.; Wu, N. C.; Fu, X. C. \& CAI, Q. H. 2008. Impacts of a small dam on riverine zooplankton. International Review of Hydrobiology 93(3):297-311. 